\title{
Reconstruction of Achilles region defects: a single-center experience
}

\author{
Cengiz Eser ${ }^{1}$, Ozgün İlke Karagöz Ceylan ${ }^{1}$, Eyuphan Gencel ${ }^{1}$, Ibrahim Tabakan ${ }^{1}$, Ömer \\ Kokaçya $^{1}$, and Metin Yavuz ${ }^{1}$ \\ ${ }^{1}$ Cukurova University Faculty of Medicine
}

February 5, 2021

\begin{abstract}
Background: Reconstruction of Achilles tendon and the overlying tissue defects is a challenging undertaking. The spectrum of available repair methods range from secondary healing to use of free flaps. Method: In this study, we retrospectively evaluated 14 patients who underwent reconstruction of Achilles region defect between 2016 and 2019 at a single center. Results: Reconstructions were performed with secondary healing $(n=2)$, negative pressure wound therapy and skin grafting $(n=2)$, free flaps $(n=6)$, and local and distant flaps $(n=4)$. Satisfactory aesthetic and functional outcomes were achieved in all patients. One patient developed partial skin graft loss. Marginal necrosis occurred in one of the local flaps. Wound dehiscence and flap retraction occurred in one of the free (superficial circumflex iliac artery perforator) flaps. One patient undergoing reconstruction with ulnar artery perforator flap developed intraoperative atrial fibrillation; the operation was terminated and reconstruction completed with skin grafting. Conclusion: Orthoplastic reconstruction should be kept in mind for Achilles tendon defects. Use of special digital imaging techniques facilitates flap surgery and helps minimize the risk of flap complications. Conventional approaches are suitable for shallow small skin lesions. Local flaps are good options for deeper skin defects owing to superior aesthetic outcomes. Superthin free flaps offer a distinct advantage in skillful hands. Use of multi-content free chimeric flaps for reconstruction of complex defects facilitates better anatomical repair. Cross leg or flow-through flaps may be considered in patients with compromised distal circulation. Selection of the most reliable approach for Achilles reconstruction is a key imperative to achieve favorable outcomes.
\end{abstract}

\section{Introduction}

Reconstruction of defects of Achilles tendon and overlying tissues is a challenging undertaking ${ }^{1,2}$. These defects are typically caused by trauma, infection, or excision of tumors ${ }^{3}$. The relatively poor blood perfusion and the thin overlying skin renders this area vulnerable to trauma and complications ${ }^{4}$. Several different approaches are used for reconstruction of the Achilles region depending upon the mechanism of trauma and individual factors. The type of reconstruction is determined based on several factors including the length of achilles tendon defect, the extent of loss of overlying tissues, and the general health of the patient. The spectrum of repair methods range from secondary healing to use of free flaps ${ }^{5-9}$. Owing to the challenging nature of Achilles reconstruction, selection of the most reliable option is a key imperative to achieve favorable outcomes.

In the contemporary literature, there is no clear consensus on the optimal approach for reconstruction of defects of the Achilles tendon and the overlying tissues. In this study, we describe our treatment methods for repair of Achilles region defects and evaluated the outcomes of Achilles region reconstruction in a cohort of patients treated at a single center over a 4 -year period.

\section{Patients and methods}


This study was conducted in accordance with the Helsinki Declaration after the approval of the local ethics committee. Fourteen patients (four female and ten male) who underwent surgical reconstruction of Achilles region defect between 2016 and 2019 were retrospectively evaluated. The mean age of patients was 39 years. If required, surgical debridement was performed prior to final reconstruction. The size of skin-soft tissue loss, length of the Achilles tendon defect, exposure of vital structures (bone, tendon, vessel, nerve, joint, or foreign material), sensation, motor function, and perfusion were evaluated and abnormal findings noted. Patients were operated under local or general anesthesia depending on operative needs. All interventions were performed by the same team. Details pertaining to patient demographics, etiology, size/content of the defect, reconstruction, and complications/reoperations are summarized in Table 1.

\section{Reconstruction with secondary healing and split thickness skin graft}

In secondary healed cases, medical dressings were applied once a day (chlorhexidine acetate $0.5 \% \mathrm{w} / \mathrm{w}$ tulle gras) taking into account the rules of asepsis and antisepsis. Negative pressure wound therapy (NPWT) was performed with $80 \mathrm{~mm} \mathrm{Hg}$ continuous mode. In patients who underwent skin grafting, split thickness skin grafts (STSGs) were obtained with an electric dermatome under general anesthesia. After grafting, bolster dressing was applied onto the graft and short leg splint was used for seven days. Bolster dressing was removed three days after the application and wound care continued with skin pomade including $2 \%$ Mupirocin.

\section{Reconstruction with local and free flaps}

Local rotation flaps were harvested based on preoperatively identified perforator using handheld Doppler or angiography. We used superselective angiography (SSA) for detection of specific peroneal perforator (Fig. 1). The pedicled hatchet shaped flaps were harvested based on posterior tibial artery perforasomes zone, as described by Abraham et al (Fig. 2) ${ }^{10}$. Cross-leg reverse sural flap was performed as described in our previous study ${ }^{11}$. Anastomosis of free medial sural artery perforator (MSAP) (Fig. 3), superficial circumflex iliac artery perforator (SCIP) (Fig. 4) and anterolateral thigh (ALT) flaps were performed in the end-to-side fashion; the cross-leg free latissimus dorsi (LD) and anterolateral thigh-tensor fascia lata (ALTTFL) flaps (Fig. 5) were anastomosed to the posterior tibial vascular system in a flow-through fashion. All patients scheduled for free flap reconstruction underwent preoperative computed tomography (CT) or magnetic resonance angiography to identify the recipient vessels and the vascular pedicle. Peroperative Indocyanine Green Angiography (ICGA) was performed in cases with suspect free or local flap circulation. In patients who underwent local or free flap repair, short leg splint was applied for postoperative seven days. In patients who received cross-leg flaps, postoperative stabilization was achieved by external fixator or cast splints. Pedicles were cut on day ten after the first session. All patients were followed up at 1 st and 6 th postoperative months.

\section{Results}

All patients showed satisfactory appearance, color, and texture of the repaired area. Moreover, all patients exhibited satisfactory functional recovery of the injured limb. None of the secondary healed cases developed any wound-related problems. Partial loss of STSG was observed in one patient owing to non-compliance with immobilization. In one of the local flaps, marginal necrosis occurred at the location determined with ICGA (Case 11). Wound dehiscence and flap retraction occurred in one of the free flaps (SCIP) due to excessive mobilization of the patient. The problem was solved by flap advancement and secondary suture (Fig. 4).

One of the patients in whom ulnar artery perforator flap was used developed atrial fibrillation during the surgery (Case 8). The operation was terminated due to the emergency and reconstruction completed with NPWT and STSG.

\section{Discussion}

Achilles region reconstruction is one of the most challenging topics of lower limb repair for plastic surgeons. The Achilles tendon is the strongest tendon of the body and has a very thin and supple overlying skin. The tibial neurovascular bundle is located just medial to the Achilles tendon. Several factors should be 
carefully evaluated to ensure successful outcomes of reconstruction; these include age of the patient, exposed vital structures, size of the defect, comorbid diseases, presence of scars, history of previous surgery, infection, medications, smoking, and patient expectations. ${ }^{1,2}$ During reconstruction in this region, neurovascular bundle repair and tendon-skin-soft tissue reconstruction can be performed together or sequentally, as per need. In the presence of a ruptured, necrotic or weakened tendon, reconstruction should be performed concomitantly with soft tissue-skin repair. Therefore, the orthoplastic approach is neccessary for such repairs.

According to the meta-analysis and algorithm developed by Hackenberg et al. ${ }^{9}$, primary tendon suturation is suitable for repair of defects sized $<2 \mathrm{~cm}$; V-Y tendinoplasty, tendon transfer, tendon rotation, plantaris, flexor hallucis longus, peroneus brevis and flexor digitorum longus tendon plasties and combinations can be applied for defects ranging from $2-5 \mathrm{~cm}$ in size. For Achilles defects sized $6-10 \mathrm{~cm}$, free tendon/muscle transplantations, fascia replacement plasties, allografts, or synthetic materials can be used. Several surgical interventions have been described for reconstruction of soft tissue defects, ranging from secondary skin healing to use of free flaps and amputation; the choice depends upon exposure of vital structures and tissue vascularity. ${ }^{12,13}$ According to Monnere et al. ${ }^{14}$, adequate neo-Achilles tendon can be formed with skin-soft tissue reconstruction without Achilles tendon repair. However, according to a meta-analysis by Ochen et al. ${ }^{15}$, operative treatment is associated with reduced rate of re-rupture of the Achilles tendon. In our opinion, it seems reasonable to perform operative treatment for Achilles tendon rupture.

In the present study, three patients had injuries in the form of skin abrasions. Secondary healing was achieved by medical dressings performed in the outpatient setting. Ankle foot orthoses were used for one week in these patients with the ankle in the neutral zero position to improve wound healing. At the end of one week, full motion was allowed with open dressing. Secondary healing is beneficial for clean Achilles wounds with small overlying skin defects. Repta et al. ${ }^{16}$ employed STSG following NPWT therapy for repair of tendon exposure and skin defect in patients with peripheral vascular disease. We also achieved satisfactory results with NPWT plus STSG treatment in two patients with diabetes mellitus and in one patient in whom the free flap operation had to be stopped due to peroperative atrial fibrillation (Case 8). However, prolonged hospitalization and graft adaptation problems are some of the drawbacks of this approach. Application of NPWT plus STSG treatment is particularly appropriate for patients with small skin defects, those with compromised vascular supply, or for whom free flaps cannot be applied. Although conventional methods such as secondary healing, NPWT, and skin grafts are preferred in some patients, development of infection in this region is liable to impair epithelialization and graft adaptation. In addition, it is difficult to achieve long-term stable coverage with conventional treatment due to chronic shoe friction.

Use of local flap is another option; however, use of local flap around the Achilles region entails a certain degree of risk. Distal lower extremity is a relatively dangerous part of the body for use of local flaps. Owing to this issue, perforator or perforator based flaps have been inreasingly used in recent years ${ }^{17,18}$. We performed perforator based flap reconstructions for shallow small skin defects. Use of local flap not only helps maintain the color but also allows for shallow skin replacement. The main problem in this area is to find a reliable perforator for flap nourishment. For this purpose, we relied on Mohan's hot spot theory ${ }^{19}$ and performed superselective angiography to identify the reliable perforator located nearest to the defect.

On the other hand, in terms of anatomic replication, the thickness of flap used in the Achillles area should be very thin. Currently, supermicrosurgical techniques allow for repair of shallow skin-soft tissue defect repairs with use of super-thin free flaps by skilled operators. Suprafascial flap dissection, perforator dissection, and perforator to perforator anastomosis are main challenges in supermicrosurgery. Using this principle, Hong et al. ${ }^{20}$ have described foot and Achilles repairs with use of super-thin gluteal perforator flaps and posterior interosseous perforator flaps. Since super-thin free flaps contain only superficial subcutaneous tissue and skin, these might be suitable alternatives for reconstruction of skin overlying the Achilles tendon. These flaps can be harvested and anastomosed based on perforator vessels without causing any damage to the main vessels in the donor and recipient areas ${ }^{21}$. While the aforementioned repair techniques require a very high level of surgical skill and instrumentation, donor area scars (gluteal and dorsal forearm) may not be preferred in some patients. In our study, free SCIP and MSAP flaps were used for this area in two patients. 
The main purpose of SCIP flap application was to create a thin layer where the shoe is in contact with the Achilles region. The MSAP flap was applied to adhere to the principle of not changing the position of the patient during surgery and using a thin flap again. The main drawback with the use of MSAP flap is that pedicle dissection is relatively difficult. However, appropriate postoperative results were obtained with both flaps.

As mentioned earlier, concomitant repair of soft tissue and tendon can be performed in the Achilles region. Lin et al. ${ }^{22}$ performed skin-soft tissue reconstruction with posterior tibial perforator flap while repairing the Achilles tendon with free fascia lata graft. They mentioned that this approach can be used safely, especially in children. Although, this approach offers the advantage of single-stage surgery and avoidance of free flap complications in children, a more anatomical repair may be preferred with single-session composite flaps in such cases. Li et al. ${ }^{23}$ performed NPWT therapy and free flap surgery following prompt debridement, and achieved satisfactory results in children with mangled extremities. Dobke et al. ${ }^{24}$ used the free temporopatietal fascia flap for soft tissue repair and the galeal extension of the fascia for Achilles tendon reconstruction. Although they achieved long-term stable tendon repair, a long scar in the scalp, especially in males, can be considered as a major drawback of this method. In addition to previous options, the number of multiple donor areas can be reduced to a single area by using chimeric free flaps. Wei et al. ${ }^{25}$ used composite free groin flaps for complex Achilles region reconstructions. In addition to tendon reconstruction, they also performed calcaneal bone and skin-soft tissue reconstruction. While the main advantage of the mentioned flap is their thinness and composite nature, the long-term risk of abdominal hernia can be considered as a disadvantage. Deiler et al. ${ }^{5}$ performed reconstruction of complex Achilles region defects with free composite TFL flap. The fascial component of the flap was used for Achilles tendon reconstruction, while the skin portion was used for reconstruction of the overlying tissue. The disadvantage of this approach is that it may require debulking for good aesthetic results, especially in patients who have thick skin-subcutaneous tissue on the lateral aspect of thigh.

The technique of flow-through repair was first described by Soutar et al. ${ }^{26}$ in 1983 . Ever since, this technique has been used to protect the circulation in the distal extremity, especially in traumatic complicated lower extremity defects. Several reports have described the use of this approach for the reestablishment of circulation in the extremity and repair of complicated lower limb defects. Koshima et al. ${ }^{27}$ used the thin LD perforator flap, Özkan et al. ${ }^{23}$ used the radial forearm flap, and Yang Z et al. ${ }^{24}$ used the ALT flap in flow-through fashion. However, significant scarring and seroma problems in the donor area may restrict the use of these flaps.

Especially in single artery extremities, without harming the remainder artery, cross-leg free flow-through, cross-leg pedicled or cross-leg flow-through flaps can be used as alternatives to flow-through free flaps ${ }^{28-30}$. In the present study, we used cross-leg reverse sural artery flap (CLRSAF) and cross flow-through LD (CFTLD) flap in order to protect the exposed Achilles tendon. We obtained satisfactory resuls with both flaps; however, postoperative awkward position is the main drawback of this approach. Instead of a free flap surgery, the main reasons for using CLRSAF were previous operations in dorsal leg and single artery extremity. Circulation of the CLFTLD flap was preferred from the opposite extremity because the patient was in the subacute traumatic period according to Godina's timing of microvascular reconstruction, and was exposed to electrical burns. Both reasons may cause thrombotic events after free flap surgeries. ${ }^{31,32}$

In addition to use of flow-through flaps, repairing the affected nerve in the distal limb complex defects is of special importance. Agarwal et al. ${ }^{33}$ transferred saphenous nerve to the posterior tibial nerve, which helped restored protective sensations in the heel. One of the patients in the present study had a complex defect involving the Achilles tendon, posterior tibial artery, vein, and nerve. The tendon and vessels were reconstructed with composite flow-through ALT-TFL flap while the posterior tibial nerve was grafted with the contralateral proximal sural nerve graft. This was because the patient's distal sural nerve had burned and the proximal healthy sural nerve was nonfunctional. The patient was able to walk two months after the operation; EMG performed one year after the operation showed restoration of protective sensation in the heel. Using this method, the three main vital structures and the skin were reconstructed in a single operation. 
Pre- and postoperative imaging is very important in the context of complex repairs. In the present study, vascular structures were evaluated preoperatively with color Doppler, CT, or MRI angiography. While planning the local flaps, we were inspired by the perforator zones and hot spots described by Taylor, Abraham, Saint Cyr, and Mohan. Most hot spots can be found adjacent to joints and at the midpoint between two joints in the extremities; however, in the trunk, the perforators are clustered parallel to the posterior and anterior midline, and in the midaxillary regions ${ }^{10,19,34,35}$. We performed superselective digital substraction angiography ${ }^{36}$ (SDSA) in one patient who had a small skin defect over the Achilles tendon. SDSA helped identify the stereotaxic location and direction of the perforator located nearest to the defect (Fig. 1b). Finally, similar to an axial flap, we harvested a perforator based flap according to the course of the perforating vessel, and avoided the risk of necrosis. Moreover, this approach complied with the "like to like reconstruction' principle. We believe that this method allows for harvesting of reliable pedicled flaps and application of 'like to like repair' even in the coldspot regions specified by Mohan et al ${ }^{19}$. Of course, high radiation dose is the most important drawback of this method. IGA, which is frequently used to evaluate the viability of mastectomy skin flaps, has recently been applied for assessment of skin flaps prior to their transfer ${ }^{37,38}$. IGA helps identify the viable portion of the flap and the location of the nutrient vessels. In our study, we also performed IGA for the perforator flap harvesting and free flap follow-up. Use of IGA helped decrease the flap dissection time, avoided dissection errors, and decreased the risk of flap necrosis.

The main limitations of this study was the small number of patients. Although lower leg injuries are frequently encountered in clinical practice, the focus of this study was specifically on the Achilles region, and not the whole lower limb.

\section{Conclusion}

Reconstruction of the Achilles tendon and the overlying skin necessitates in-depth knowledge of the lower leg reconstructive armamantorium. Orthoplastic reconstruction should keep in mind for tendon defects. Special digital imaging facilitates flap surgery and may reduce the risk of flap complications. Conventional approaches are suitable for shallow small skin lesions of the Achilles region. For deeper skin defects, local flaps are good options for good aesthetic outcomes. In addition, super-thin free flaps provided excellent results in skillful hands. For complex defects, use of multi-content free chimeric flaps for reconstruction allows for better anatomical repair. Cross-leg or flow-through flaps may be considered for Achilles repairs in patients with compromised distal circulation. Selection of the most reliable approach for Achilles reconstruction is a key imperative to achieve favorable outcomes.

Conficts of interest: None

\section{References}

1. Soons J, Rakhorst HA, Ruettermann M, Luijsterburg AJ, Bos PK, Zophel OT. Reconstruction of defects involving the Achilles tendon and local soft tissues: a quick solution for a lingering problem. Bone Joint $J$. 2015;97-B(2):215-220.

2. Zhu YL, Xu YQ, Mei LB, Wang Y, Li FB, Sun LH. Open achilles tendon defects: a 12-year study on the injury mechanisms, reconstructions, and late complications. J Reconstr Microsurg. 2014;30(8):569-580.

3. Bullocks JM, Hickey RM, Basu CB, Hollier LH, Kim JY. Single-stage reconstruction of Achilles tendon injuries and distal lower extremity soft tissue defects with the reverse sural fasciocutaneous flap. J Plast Reconstr Aesthet Surg. 2008;61(5):566-572.

4. Agrawal NK, Bhattacharya V. V-Y gastrocnemius muscle slide with turnover fascial flap for compound Achilles defects: a simple solution.J Plast Reconstr Aesthet Surg. 2010;63(4):e406-410.

5. Deiler S, Pfadenhauer A, Widmann J, Stutzle H, Kanz KG, Stock W. Tensor fasciae latae perforator flap for reconstruction of composite Achilles tendon defects with skin and vascularized fascia. Plast Reconstr Surg. 2000;106(2):342-349. 
6. Bertasi G, Cole W, Samsell B, Qin X, Moore M. Biological incorporation of human acellular dermal matrix used in Achilles tendon repair. Cell Tissue Bank. 2017;18(3):403-411.

7. Cole W, Samsell B, Moore MA. Achilles Tendon Augmented Repair Using Human Acellular Dermal Matrix: A Case Series. J Foot Ankle Surg.2018;57(6):1225-1229.

8. Egger AC, Berkowitz MJ. Achilles tendon injuries. Curr Rev Musculoskelet Med. 2017;10(1):72-80.

9. Hackenberg RK, Tager S, Prangenberg C, Kabir K, Welle K. Reconstruction of Complicated Achilles Tendon Ruptures with Soft Tissue Defects - a Systematic Overview and Development of a Treatment Algorithm. Z Orthop Unfall. 2020.

10. Abraham JT, Saint-Cyr M. Keystone and Pedicle Perforator Flaps in Reconstructive Surgery: New Modifications and Applications. Clin Plast Surg. 2017;44(2):385-402.

11. Eser C, Kesiktas E, Gencel E, Aslaner EE, Yavuz M. An alternative method to free flap for distal leg and foot defects due to electrical burn injury: distally based cross-leg sural flap. Ulus Travma Acil Cerrahi Derg. 2016;22(1):46-51.

12. Veronesi F, Borsari V, Contartese D, Xian J, Baldini N, Fini M. The clinical strategies for tendon repair with biomaterials: A review on rotator cuff and Achilles tendons. J Biomed Mater Res B Appl Biomater. 2020;108(5):1826-1843.

13. Wegrzyn J, Luciani JF, Philippot R, Brunet-Guedj E, Moyen B, Besse JL. Chronic Achilles tendon rupture reconstruction using a modified flexor hallucis longus transfer. Int Orthop.2010;34(8):1187-1192.

14. Monnerie C, Le Nen D, Hu W, Letissier H, Kerfant N. [Treatment of necrosis of Achilles tendon without tendon reconstruction: About four cases]. Ann Chir Plast Esthet. 2019;64(2):208-214.

15. Ochen Y, Beks RB, van Heijl M, et al. Operative treatment versus nonoperative treatment of Achilles tendon ruptures: systematic review and meta-analysis. BMJ. 2019;364:k5120.

16. Repta R, Ford R, Hoberman L, Rechner B. The use of negative-pressure therapy and skin grafting in the treatment of soft-tissue defects over the Achilles tendon. Ann Plast Surg. 2005;55(4):367-370.

17. Al-Mousawi A, Sanese G, Baljer B, et al. Use of the Keystone Perforator Island Flap in the treatment of chronic lower extremity wounds complicated by osteomyelitis. Injury. 2020;51(3):744-749.

18. Li P, Li Z, Shen G. Distally Based Posterior Tibial Artery Perforator Flaps for Reconstruction of the Defects in Achilles Region.Ann Plast Surg. 2019;83(4):452-454.

19. Mohan AT, Sur YJ, Zhu L, et al. The Concepts of Propeller, Perforator, Keystone, and Other Local Flaps and Their Role in the Evolution of Reconstruction. Plast Reconstr Surg.2016;138(4):710e-729e.

20. Hong JP. The use of supermicrosurgery in lower extremity reconstruction: the next step in evolution. Plast Reconstr Surg.2009;123(1):230-235.

21. Suh HS, Oh TS, Lee HS, et al. A New Approach for Reconstruction of Diabetic Foot Wounds Using the Angiosome and Supermicrosurgery Concept.Plast Reconstr Surg. 2016;138(4):702e-709e.

22. Lin CZ, Xia X, Wang H, Liu DX. Surgical reconstruction of the fascia lata and posterior tibial artery perforator flap to treat children with simultaneous injury to the Achilles tendon and heel skin. Medicine (Baltimore). 2018;97(6):e9834.

23. Li RG, Yu B, Wang G, et al. Sequential therapy of vacuum sealing drainage and free-flap transplantation for children with extensive soft-tissue defects below the knee in the extremities. Injury.2012;43(6):822-828.

24. Dobke M, Suliman A, Mackert GA, Herrera FA, Singer R, Nelson J. Single-Stage Reconstruction of Achilles Tendon and Overlying Tissue With the Extended Temporoparietal Fasciagaleal Flap-23-Year FollowUp and the Review of the Literature. Ann Plast Surg. 2016;76 Suppl 3:S165-170. 
25. Wei FC, Chen HC, Chuang CC, Noordhoff MS. Reconstruction of Achilles tendon and calcaneus defects with skin-aponeurosis-bone composite free tissue from the groin region. Plast Reconstr Surg.1988;81(4):579589.

26. Soutar DS, Scheker LR, Tanner NS, McGregor IA. The radial forearm flap: a versatile method for intra-oral reconstruction. Br J Plast Surg. 1983;36(1):1-8.

27. Koshima I, Saisho H, Kawada S, Hamanaka T, Umeda N, Moriguchi T. Flow-through thin latissimus dorsi perforator flap for repair of soft-tissue defects in the legs. Plast Reconstr Surg.1999;103(5):1483-1490.

28. Gencel E, Eser C, Kesiktas E, Tabakan I, Yavuz M. A cross flow-through pedicle free latissimus dorsi flap for high voltage electrical burns. Burns. 2016;42(4):e55-60.

29. Atiyeh BS, Al-Amm CA, El-Musa KA, Sawwaf AW, Musharafieh RS. Distally based sural fasciocutaneous cross-leg flap: a new application of an old procedure. Plast Reconstr Surg. 2003;111(4):1470-1474.

30. Liang W, Tan BK. Use of the cross-leg distally based sural artery flap for the reconstruction of complex lower extremity defects.Arch Plast Surg. 2019;46(3):255-261.

31. Godina M. Early microsurgical reconstruction of complex trauma of the extremities. Plast Reconstr Surg. 1986;78(3):285-292.

32. Lee ZH, Stranix JT, Rifkin WJ, et al. Timing of Microsurgical Reconstruction in Lower Extremity Trauma: An Update of the Godina Paradigm. Plast Reconstr Surg. 2019;144(3):759-767.

33. Agarwal P, Shukla P, Sharma D. Saphenous nerve transfer: A new approach to restore sensation of the sole. J Plast Reconstr Aesthet Surg. 2018;71(12):1704-1710.

34. Saint-Cyr M, Wong C, Schaverien M, Mojallal A, Rohrich RJ. The perforasome theory: vascular anatomy and clinical implications. Plast Reconstr Surg. 2009;124(5):1529-1544.

35. Taylor GI, Palmer JH. The vascular territories (angiosomes) of the body: experimental study and clinical applications. Br J Plast Surg. 1987;40(2):113-141.

36. Hoffmann JC, Minkin J, Primiano N, Yun J, Eweka A. Use of a steerable microcatheter during superselective angiography: impact on radiation exposure and procedural efficiency. CVIR Endovasc.2019;2(1):35.

37. Driessen C, Arnardottir TH, Lorenzo AR, Mani MR. How should indocyanine green dye angiography be assessed to best predict mastectomy skin flap necrosis? A systematic review. J Plast Reconstr Aesthet Surg. 2020.

38. Narushima M, Yamasoba T, Iida T, et al. Pure Skin Perforator Flaps: The Anatomical Vascularity of the Superthin Flap. Plast Reconstr Surg. 2018;142(3):351e-360e.

\section{Figure Legends:}

Figure 1: The patient whose defect was repaired with PAP based rotation flap

1. Posterior view of skin-soft tissue defect.

2. Digitally substracted view of superselective angiography of PAP. The flap was designed according to the suitable perforator located nearest to the defect.

3. The designed PAP based rotation flap

4. Two years follow-up

PAP: Peroneal artery perforator

Figure 2: Shallow Achilles overlying skin reconstruction with PTA based hatchet flap

1. Acute defect of the skin overlying the Achilles tendon

2. Harvested medially based hatched fasciocutaneous flap (yellow spots show probable location of PTA perforators) 
3. Two years follow-up

PTA: posterior tibial artery

Figure 3: Traumatic Achilles region defect reconstructed with free MSAP flap

1. Unhealed Achilles overlying skin defect

2. The harvested MSAP flap

3. Two months follow-up

MSAP: medial sural artery perforator

Figure 4: Achilles overlying skin defect reconstruction with free SCIP flap

Exposed Achilles tendon

Advanced and re-sutured SCIP flap

SCIP: superficial circumflex iliac artery perforator

Figure 5: The patient whose composite Achilles region defect were repaired with flow-through ALT-TFL chimeric flap and sural nerve graft.

1. Medial view of perforated and burnt lower extremity due to hot metal injury

2. Peroperative view of the posterior tibial neurovascular defect

3. Inset of flow-through ALT-TFL chimeric flap. Note that the TFL mimics Achilles tendon, the vascular pedicle mimics the posterior tibial artery and vein, and the sural nerve graft mimics the posterior tibial nerve

4. Two years follow-up

PSPTN: Proximal stump of posterior tibial nerve; PVS: proximal vascular stump; DSPTN: distal stump of posteriot tibial nerve; DVS: distal vascular stump; ALT: anterolateral thigh; TFL: tensor fascia lata; LCFA: lateral Circumflex Femoral Artery; FTP: flow-through pedicle, NG: nerve graft

Table 1:

\section{Case}

1

2

3

4

5

6

7

8

9

10

11

12

13

14

STSG: split thickness skin graft; NPWT: negative pressure wound therapy; MSAP: medial sural artery perforator; SCIP: su 

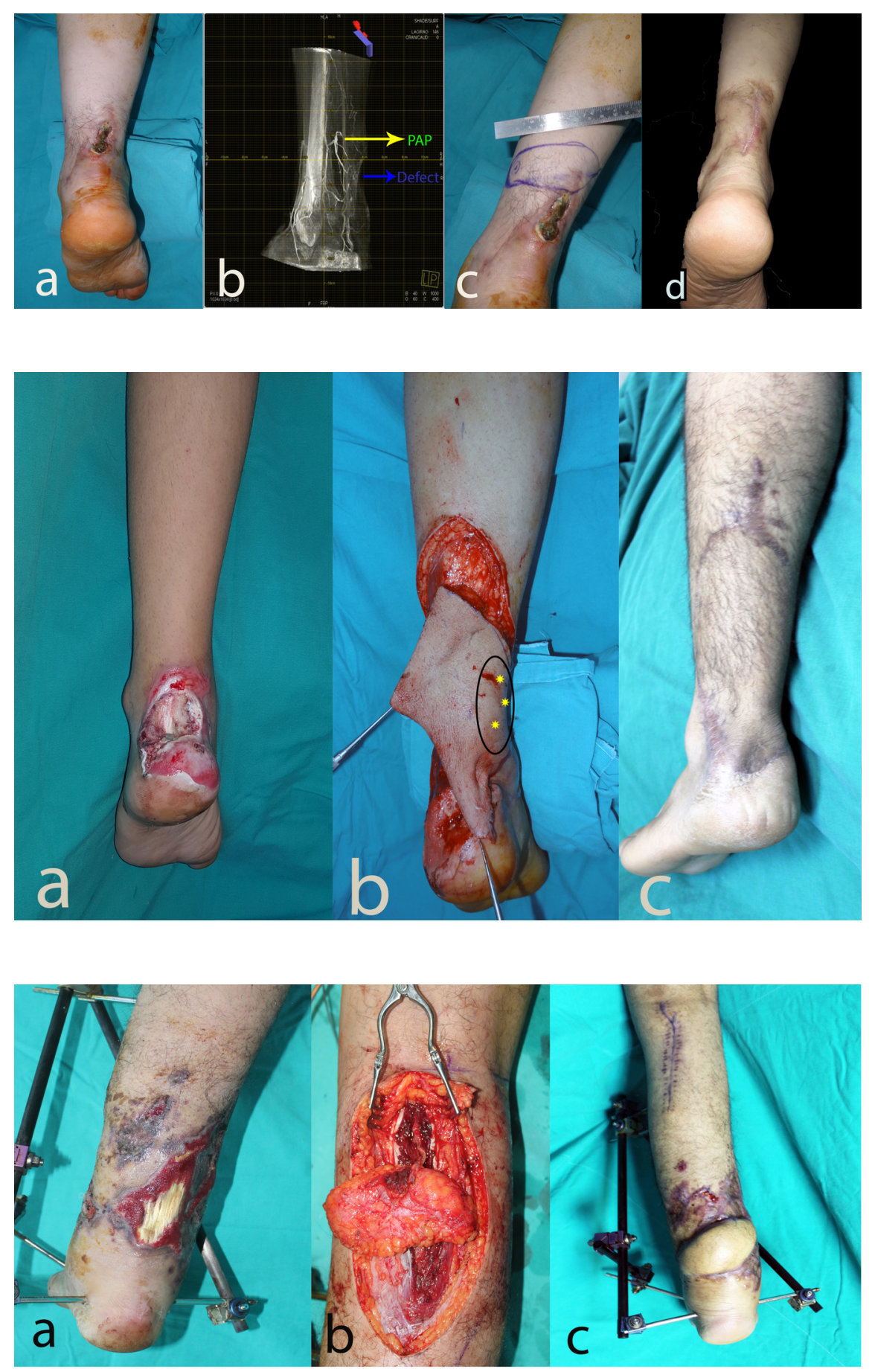

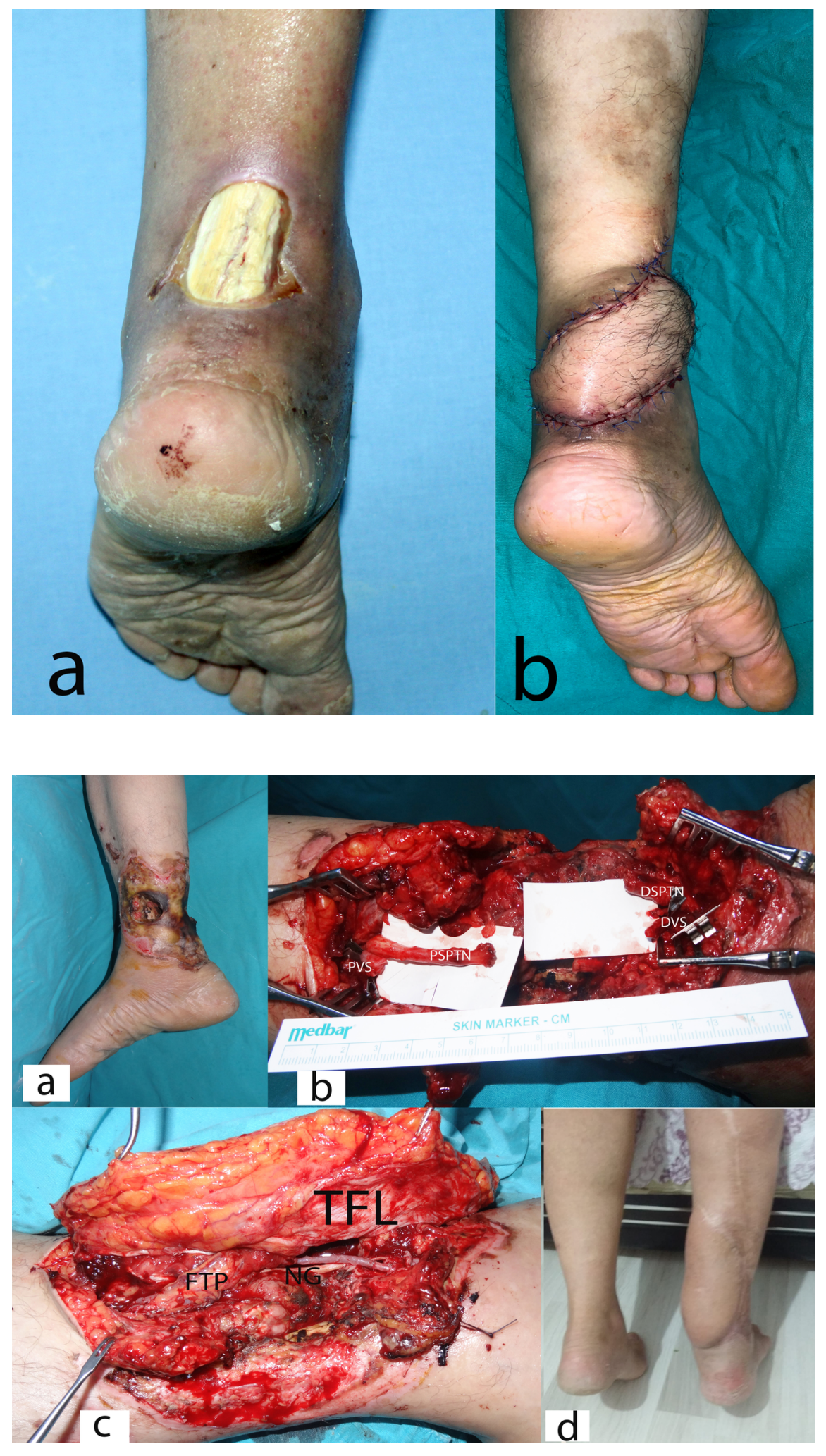